\title{
Metabolic Syndrome among Ischaemic Stroke Patients in Ghana: The Possible Role of Renin and Aldosterone
}

\author{
Francis Agyemang Yeboah', Bernard Nkum², Bright Amankwaa ${ }^{1}$, Benjamin Ackon Eghan Jr. ${ }^{2}$, \\ Emmanuel Acheampong1, Paul Nsiah ${ }^{3}$, Perditer Okyere ${ }^{2}$, Lawrence Owusu4, \\ Emmanuella Batu Nsenba ${ }^{1}$, Enoch Odame Anto ${ }^{1,5}$
}

${ }^{1}$ Department of Molecular Medicine, School of Medical Sciences, Kwame Nkrumah University of Science and Technology (KNUST), Kumasi, Ghana

${ }^{2}$ Medicine Department, School of Medical Sciences, Kwame Nkrumah University of Science and Technology (KNUST), Kumasi, Ghana

${ }^{3}$ Chemical Pathology Department, School of Medical Sciences, University of Cape Coast (UCC), Cape-Coast, Ghana

${ }^{4}$ Biochemistry and Biotechnology Department, College of Science, Kwame Nkrumah University of Science and

Technology (KNUST), Kumasi, Ghana

${ }^{5}$ School of Medical and Health Science, Edith Cowan University, Perth, Australia

Email: ^brytespiro@gmail.com

How to cite this paper: Yeboah, F.A., Nkum, B., Amankwaa, B., Eghan Jr., B.A., Acheampong, E., Nsiah, P., Okyere, P., Owusu, L., Nsenba, E.B. and Anto, E.O. (2017) Metabolic Syndrome among Ischaemic Stroke Patients in Ghana: The Possible Role of Renin and Aldosterone. Open Access Library Journal, 4: e3952.

https://doi.org/10.4236/oalib.1103952

Received: September 19, 2017

Accepted: October 15, 2017

Published: October 18, 2017

Copyright (C) 2017 by authors and Open Access Library Inc.

This work is licensed under the Creative Commons Attribution International License (CC BY 4.0).

http://creativecommons.org/licenses/by/4.0/ (c) (†) Open Access

\begin{abstract}
Background: Both metabolic syndrome (MetSyn) and the Renin Angiotensin Aldosterone System (RAAS) are predictors of adverse outcomes in stroke patients. This study aimed at evaluating the association between RAAS and MetSyn among ischaemic stroke subjects in a tertiary hospital of Ghana from September 2015 to June 2016. Methodology: The study purposively recruited 252 Ghanaians comprising 132 ischaemic stroke patients and 120 apparently healthy control subjects. The participants were subjected to measurements of plasma renin, serum aldosterone, lipid profile, anthropometries and blood pressure. Results: MetSyn prevalence among the stroke subjects compared to the controls were $50.0 \%$ vs $8.3 \%$ (NCEP/ATP III), $71.2 \%$ vs 9.2 (IDF) and $71.2 \%$ vs $6.7 \%$ (H_MS). Both renin and aldosterone were significantly ( $p<$ $0.05)$ higher in the ischaemic stroke subjects $[(1.8 \pm 0.1$ vs $1.4 \pm 0.1) \mathrm{pg} / \mathrm{ml}$ and $(2.9 \pm 0.2$ vs $2.3 \pm 0.2) \mathrm{pg} / \mathrm{ml}$ respectively]. Adjusting for age, gender, diabetes status and hypertension, third tertile $\left(\mathrm{T}_{3}\right)$ aldosterone $(\mathrm{aOR}=2.7, p=$ $0.008)$, obesity $(\mathrm{aOR}=11.7, p=0.004)$ and high triglyceride $(\mathrm{aOR}=5.3, p<$ $0.001)$ but not renin ( $\mathrm{aOR}=6.1, p=0.742)$ were independently associated with increasing odds of metabolic syndrome. Moreover, there was a significant $(p<0.050)$ correlation between plasma renin and waist circumference
\end{abstract}


(WC) $(\mathrm{r}=0.493)$ and high density lipoprotein (HDL) $(\mathrm{r}=-0.319)$. Significant $(p<0.050)$ correlations also existed between serum aldosterone and WC $(\mathrm{r}=$ $0.588)$, waist-to-height ratio (WHR) $(\mathrm{r}=0.503)$, body mass index (BMI) $(\mathrm{r}=$ $0.691)$, HDL $(r=-0.317)$, total cholesterol $(\mathrm{r}=0.678)$ and triglyceride $(\mathrm{r}=$ 0.439). Conclusion: Aldosterone and not renin could play significant role in the pathophysiology of metabolic syndrome in ischaemic stroke. These findings underpin the observations that aldosterone is associated with several cardiovascular risk factors and may exacerbate metabolic defects in people with ischaemic stroke. Adequate aldosterone blockade could therefore mitigate the development and progression of metabolic syndrome in ischaemic stroke subjects.

\section{Subject Areas}

Internal Medicine, Molecular Biology

\section{Keywords}

Renin, Aldosterone, Metabolic Syndrome, Ischaemic Stroke

\section{Introduction}

Stroke is an increasing public health concern globally and its victims are particularly susceptible to cardiometabolic complications including dyslipidaemia, diabetes and obesity [1]. Metabolic syndrome (MetSyn) on the other hand is a term coined for the clustering of cardiovascular risk factors including diabetes mellitus, hypertriglyceridaemia, central obesity, increased low density lipoprotein cholesterol (LDL-C) and decreased high density lipoprotein cholesterol (HDL-C) [1]. MetSyn has been shown to increase risk for stroke and other cardiovascular diseases [2]. According to [3] [4], metSyn confers 5-fold risk for type 2 diabetes and 2 to 3 fold increased risk for coronary heart disease and future ischaemic stroke [3] [4]. Prevalence of MetSyn is estimated to range from $<10 \%$ to as high as $84 \%$ depending on the region and underlying condition of study [5]. Previous studies have indicated high prevalence of metabolic syndrome particularly among ischaemic stroke subjects [1] [6].

In a case-control study among Iranians, $62 \%$ stroke subjects compared to $34 \%$ normal controls had metabolic syndrome. Also, in a case-control study involving 100 Ghanaian subjects with various cardiovascular diseases (stroke, coronary heart disease, peripheral arterial disease or heart failure) the prevalence of MetSyn was $54 \%[7]$.

Whereas the cause of metabolic syndrome is uncertain and is believed to be multifactorial, the renin angiotensin aldosterone system (RAAS) has been found to play a significant role in its pathophysiology [1]. RAAS has been linked to components of MetSyn including obesity, dyslipidaemia, hypertension and insulin resistance [2] [8]. 
The RAAS could be linked to cardiometabolic syndrome through possible interactions with atherogenic factors including vascular smooth muscle cells, mitogen-activated protein (MAP) kinase, intercellular adhesion molecule and oxidative stress (ICAM) 1 [9] [10] [11].

Studies suggest that mineralocorticoid receptor (MR) antagonists could be important therapeutic agents in mitigating MetSyn and its complications [12] [13].

However, data supporting the use of MR antagonists in ischaemic patients with the aim of preventing further metabolic defect is limited. There remains a paucity of data examining the relationship between the RAAS and the MetSyn in particularly ischaemic stroke. A better understanding of the association of RAAS and MetSyn may help in the management of ischaemic stroke subjects and thus minimize, if not entirely eradicate mortality from obesity, diabetes and hypertension [14]. Additionally, understanding the relationship between the RAAS and MetSyn in ischaemic strokes may help in the stratification and treatment of patients who may benefit substantially from RAAS blockade.

\section{Materials and Method}

\subsection{Study Design and Setting}

A case-control study which was carried out at the Medicine Department of Komfo Anokye Teaching Hospital (KATH) in the Ashanti Region of Ghana. Komfo Anokye Teaching Hospital is the second largest tertiary hospital in Ghana with over a thousand bed capacity and is a major referral centre that provides health services to five other regions in Ghana. The study spanned through September 2015 to June 2016. However only two months were used to recruit the stroke cases due to its acute state.

\subsection{Study Population and Subject Recruitment}

A randomized sampling technique was used to recruit a total of 252 subjects (132 cases and 120 controls). The 132 sample size was based on the monthly incidence of stroke $44(9.1 \%)$ at KATH [15]. The cases were admitted ischaemic stroke patients diagnosed with computerized tomographic scan and confirmed by a neurologist whereas apparently normal subjects without any history of CVD attending the hospital for medical check-ups served as controls. Patients with chronic kidney disease (protein-creatinine-ratio greater than 0.3 in women and $0.2 \mathrm{in} \mathrm{men} \mathrm{and/or} \mathrm{eGFR}<60 \mathrm{~mL} / \mathrm{min} / 1.73 \mathrm{~m}^{2}$ ) [16], were exempted from the study.

\subsection{Ethical Consideration}

Ethical approval for the study (CHRPE/AP/377/15), was obtained from the Committee on Human Research, Publication and Ethics of the School of Medical Sciences (SMS), Kwame Nkrumah University of Science and Technology (KNUST) as well as ethical review board of the Komfo Anokye Teaching Hospital (KATH). Informed consent was obtained from all participants and participation 
was strictly voluntary.

\subsection{Questionnaire}

Structured questionnaire was administered to determine socio-demographics and identified risk factors of hypertension such as age, gender, and alcohol consumption and family history cardiovascular diseases.

\subsection{Blood Pressure Measurement}

Blood pressure (BP) measurements were done using the Omron M5-I digital fully automatic blood pressures monitor (Omron, Japan). After subjects had sat quietly for at least ten minutes, three measurements were taken at 5 minutes interval on the left arm in a seated position, with arm supported at heart level and feet flat on the floor. Hypertension was diagnosed when the mean of the second and third blood pressure (BP) measurements was equal to or above 140/90 $\mathrm{mmHg}$ [17].

\subsection{Measurement of Anthropometric Variables}

Height to the nearest centimetre and weight to the nearest $0.1 \mathrm{~kg}$ in light clothing were measured with a wall-mounted ruler and a bathroom scale (EatSmart ESBS-07 Precision Series Tracker Digital Bathroom Scale, USA). Body mass index (BMI) was calculated as weight $(\mathrm{kg}) /$ height $^{2}\left(\mathrm{~m}^{2}\right)$. Waist circumference was measured at the midpoint between the last rib and the iliac crest with an Esprit spring-loaded measuring tape (SpringLoaded Technology USA) while the subject stood wearing light cloths. The hip circumference [18] was measured at the widest level over the greater trochanters. The waist-to-hip ratio (WHR) was calculated by dividing the waist circumference $(\mathrm{cm})$ by the hip circumference $(\mathrm{cm})$. All measurements were taken thrice and the means recorded.

\subsection{Blood Sampling, Processing and Biochemical Assays}

Fasting whole blood samples were drawn by venipuncture after 10 minutes of rest in a seated position in the morning between 6:30 and 9:30 AM. Blood was collected in EDTA tubes, maintained at $0^{\circ} \mathrm{C}-4^{\circ} \mathrm{C}$ during delivery to the laboratory, and rapidly frozen at $-80^{\circ} \mathrm{C}$ after centrifugation done at $500 \mathrm{~g}$ for $15 \mathrm{mi}$ nutes. When ready to use, frozen blood samples were thawed at room temperature $\left(25^{\circ} \mathrm{C}\right)$. Plasma renin was measured as direct active renin (DAR; picograms per milliliter) by an immunoassay kit (Biobase Biotech (Jinan) Co. Ltd). The intra-assay and inter-assay coefficients of variation of the renin assay were $<9 \%$ and $15 \%$ respectively. The normal plasma renin range was given as 5.2 to 45.6 $\mathrm{pg} / \mathrm{ml}$ in upright position [19]. Serum aldosterone was measured in $\mathrm{pg} / \mathrm{ml}$ with immunoassay kit from Biobase Biotech (Jinan) Co. Ltd. The intra-assay and inter-assay coefficients of variation of the aldosterone assay were $<9 \%$ and $15 \%$ respectively. The reference interval was given as $65 \mathrm{pg} / \mathrm{ml}-450 \mathrm{pg} / \mathrm{mL}$.

Fasting total cholesterol, high density lipoprotein (HDLc) and triglyceride 
concentrations were determined by standard enzymatic methods on a Cobas C11 analyzer (Roche Diagnostics, UK). Low density lipoprotein cholesterol (LDL-C) was calculated using the Frederickson-Friedwald's formula; LDL-C = TC- TG/2-HDL-C [16].

\subsection{Estimated Glomerular Filtration Rate and Diagnosis of Chronic Kidney Disease}

Estimated glomerular filtration rate (eGFR) and was derived based on participants' serum creatinine, gender, age and race with the Chronic Kidney Disease Epidemiology Collaboration (CKD-EPI) equation [20]. CKD was defined by proteinuria (protein-creatinine-ratio greater than 0.3 in women and 0.2 in men) and/or eGFR $<60 \mathrm{~mL} / \mathrm{min} / 1.73 \mathrm{~m}^{2}$ [16].

\subsection{Metabolic Syndrome Definition}

Three metabolic syndrome definitions were defined as shown in Table 1.

\section{Data Analysis}

Normality of all continuous variables was tested using the D'Agostino-Pearson omnibus normality test. All non-parametric variables were normalized by log transformation before analysis. Continuous variables were expressed as mean \pm Standard deviation (SD) and median (inter-quartile range) where appropriate. Categorical variables were expressed as frequency (n) and percentages (\%). Associations of biochemical and clinical profile with metabolic syndrome among hypertensive were done using chi-square $\left(\chi^{2}\right)$ tests or Fisher exact tests where appropriate. Three logistic regression models (A, B and C) were done with metabolic syndrome defined by $\mathrm{H}_{-}$MS criteria as dependent variable. Model A was unadjusted, model B was adjusted for age and gender whereas model $\mathrm{C}$ was adjusted for age, gender and hypertension. In all the models, lifestyle characteristics, BMI, lipid profile and RAAS parameters served as predictor variables. $P$-value less than 0.05 was considered statistically significant for all analysis. IBM Statistical Package for the Social Sciences (SPSS) version 20.00 (SPSS Inc, Chicago, USA).

Table 1. Definition of metabolic syndrome.

\begin{tabular}{ccccccc}
\hline Mets Criteria & WC/CO & TG & HDL-C & FBG & Applied Factors & Reference \\
\hline NCEP-ATP III & $>88$ & $\geq 1.7$ & $<1.29$ & $\geq 5.6$ & Any 3 or more factors & {$[21]$} \\
IDF & $>80^{*}$ & $\geq 1.7$ & $<1.29$ & $\geq 5.6$ & WC and any 2 factors & {$[22]$} \\
H_MS & $>80$ & $\geq 1.8$ & $<1.30$ & $\geq 5.7$ & Any 3 or more factors & {$[23]$} \\
\hline
\end{tabular}

*Principal component of definition; ATP III = Adult Treatment Panel III; IDF = International Diabetes Federation; H_MS: Harmonization, IGT = impaired, glucose tolerance; impaired fasting glycaemia; T2DM

= Type 2 diabetes mellitus; $\mathrm{BMI}=$ body mass index; $\mathrm{WC}=$ waist circumference; $\mathrm{TG}=$ triglycerides; HDL-C

= high-density lipoprotein cholesterol; WC/CO: waist circumference/central obesity, WHR: waist-to-hip ratio, BMI: Body Mass Index, BP: Blood Pressure, TG: Triglycerides, HDL-C: High Density Lipoprotein Cholesterol, FBG: Fasting Blood Glucose. 


\section{Results}

The controls and stroke cases were age matched $(p=0.054)$ (Table 2). There were more males in the stroke subjects $(62.1 \%)$ compared to the controls (47.5\%) and differed significantly $(p=0.023)$. The stoke subjects had significantly higher mean plasma renin [ $(1.8 \pm 0.1) \mathrm{pg} / \mathrm{ml}, p<0.001]$, serum aldosterone $[(2.9 \pm 0.2) \mathrm{pg} / \mathrm{ml}, p<0.001)]$ and ARR [(1.1 \pm 0.2$), p<0.001]$ compared to the controls $[(1.4 \pm 0.1) \mathrm{pg} / \mathrm{ml},(2.3 \pm 0.2) \mathrm{pg} / \mathrm{ml}$ and $(0.9 \pm 0.1)]$ respectively. Additionally, the case cohort had significantly larger average waist circumference $(88.8 \pm 14.6$ vs $84.4 \pm 8.2, p=0.026) \mathrm{cm}$, waist-to-hip ratio $(0.9 \pm 0.1$ vs 0.8 $\pm 0.1, p=0.006)$ and body mass index $(27.8 \pm 4.7$ vs $23.4 \pm 5.1, p<0.001) \mathrm{kg} / \mathrm{m}^{2}$.

Per the NCEP-ATP, IDF and HMS criteria, metSyn prevalence of $8.3 \%, 9.2 \%$ and $6.7 \%$ respectively were recorded in the control subjects where as $50.0 \%$, $71.2 \%$ and $71.2 \%$ respectively were recorded in the stroke subjects. Moreover, $36.4 \%, 12.1 \%$ and $13.6 \%$ of the stroke participants per NCEP-ATP, IDF and HMS criteria respectively had 2 clusters of metabolic syndrome components.

In the Bland Altman analysis above, there was an excellent agreement between NCEP_ATP versus IDF $[$ bias $=-0.107,95 \%$ CI $(-0.545-0.759)]$, NCEP_ATP versus H_MS [bias $=-0.093,95 \% \mathrm{CI}(-0.525-0.710)]$, IDF versus H_MS [bias = $-0.346,95 \% \mathrm{CI}(-0.317-0.317)]$ (Figure 1$)$.

In the unadjusted model $\mathrm{A}$, hypertension $(\mathrm{OR}=9.2, p=0.001)$, diabetes (OR $=5.8, p<0.001)$, obesity $(\mathrm{OR}=12.5, p<0.001)$, third tertile renin $\left(\mathrm{T}_{3}\right)(\mathrm{OR}=$ 9.1, $p<0.001)$, third tertile aldosterone $\left(\mathrm{T}_{3}\right)(\mathrm{OR}=3.6, p<0.001)$ and high total cholesterol $(\mathrm{OR}=2.4, p=0.039)$ were significantly associated with metabolic syndrome compared to normotensives, non-diabetics, non-obese, lower reninaldosterone quartiles and desirable total cholesterol values respectively. However, after adjusting for age, gender, diabetes and hypertension in model C, only obesity ( $\mathrm{aOR}=11.7, p=0.004)$ and third tertile aldosterone $(\mathrm{OR}=2.7, p=$ 0.008 ) were significantly associated with increased odds of metabolic syndrome.

Whereas plasma renin moderately correlated positively and significantly with WC $(r=0.493, p<0.05)$ in metabolic syndrome subjects, it insignificantly

Bias $=0.107 ; 95 \% \mathrm{Cl}(-0.545-0.7594)$

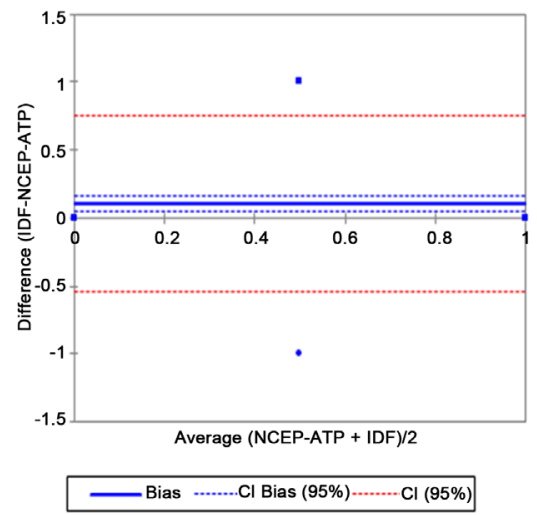

Bias $=0.0929 ; 95 \% \mathrm{Cl}(-0.525-0.7103)$

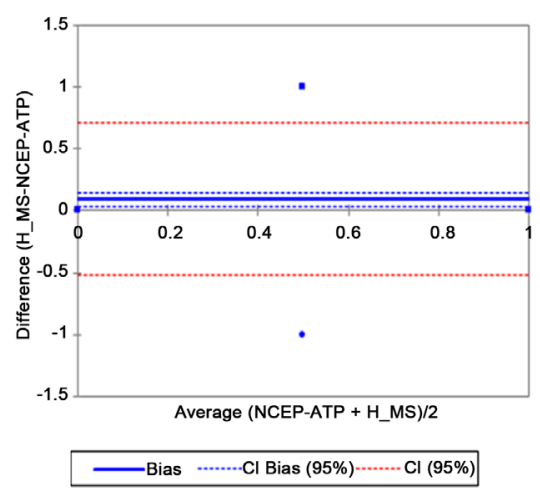

Bias $=-0.014 ; 95 \% \mathrm{Cl}(-0.346-0.317)$

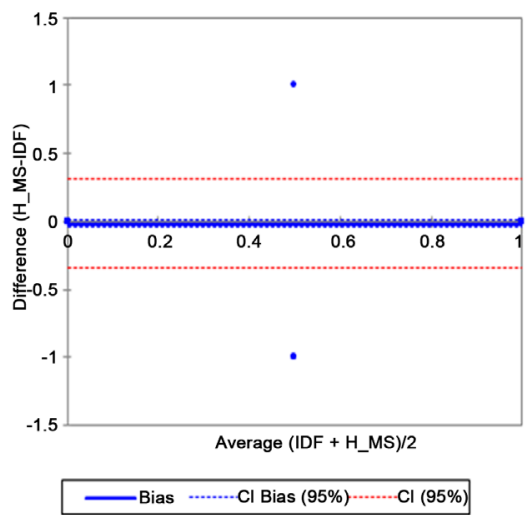

Figure 1. Degree of agreement between NCEP ATP III, IDF and H_MS definitions in diagnosing metabolic syndrome. 
Table 2. General characteristics of study participants.

\begin{tabular}{|c|c|c|c|}
\hline Parameters & Controls, n (\%) & Cases, n (\%) & $p$-value \\
\hline Age (years) & $57.5 \pm 11.1$ & $59.6 \pm 12.8$ & 0.054 \\
\hline \multicolumn{4}{|l|}{ Sex } \\
\hline Male & $57(47.5)$ & $82(62.1)$ & 0.023 \\
\hline Female & $63(52.5)$ & $50(37.9)$ & \\
\hline \multicolumn{4}{|l|}{ Anthropometry } \\
\hline $\mathrm{WC}(\mathrm{cm})$ & $84.4 \pm 8.2$ & $88.8 \pm 14.6$ & 0.026 \\
\hline $\mathrm{HC}(\mathrm{cm})$ & $102.6 \pm 10.9$ & $102.0 \pm 13.9$ & 0.784 \\
\hline WHR & $0.8 \pm 0.1$ & $0.9 \pm 0.1$ & 0.006 \\
\hline BMI $\left(\mathrm{kg} / \mathrm{m}^{2}\right)$ & $23.4 \pm 5.1$ & $27.8 \pm 4.7$ & $<0.001$ \\
\hline \multicolumn{4}{|l|}{ Haemodynamic } \\
\hline SBP $(\mathrm{mmHg})$ & $123.5 \pm 4.5$ & $168.9 \pm 20.8$ & $<0.001$ \\
\hline $\mathrm{DBP}(\mathrm{mmHg})$ & $77.1 \pm 4.3$ & $99.2 \pm 16.3$ & $<0.001$ \\
\hline $\mathrm{PP}(\mathrm{mmHg})$ & $46.4 \pm 5.1$ & $69.7 \pm 10.6$ & $<0.001$ \\
\hline MAP (mmHg) & $92.5 \pm 3.7$ & $122.4 \pm 17.2$ & $<0.001$ \\
\hline Hypertension (yes) n, (\%) & NA & $120(90.9)$ & \\
\hline Hypertension duration (years) & NA & $7.1(1.4-16.3)$ & \\
\hline \multicolumn{4}{|l|}{ Socio-demographic } \\
\hline Past alcohol status & $73(60.8)$ & $66(50.0)$ & 0.234 \\
\hline Family history of HTN & $60(50.0)$ & $36(27.2)$ & 0.009 \\
\hline Family history of CVD & $3(2.5)$ & $12(9.1)$ & 0.148 \\
\hline \multicolumn{4}{|l|}{ Biochemical Assays } \\
\hline Log renin $(\mathrm{pg} / \mathrm{ml})$ & $1.4 \pm 0.1$ & $1.8 \pm 0.1$ & $<0.001$ \\
\hline Log aldosterone (pg/ml) & $2.3 \pm 0.2$ & $2.9 \pm 0.2$ & $<0.001$ \\
\hline $\log A R R$ & $0.9 \pm 0.1$ & $1.1 \pm 0.2$ & $<0.001$ \\
\hline Total cholesterol (mmol/l) & $5.2 \pm 0.9$ & $5.5 \pm 1.4$ & 0.088 \\
\hline Triglyceride (mmol/l) & $1.5 \pm 0.7$ & $1.2 \pm 0.4$ & 0.007 \\
\hline HDL-cholesterol (mmol/l) & $1.8 \pm 0.3$ & $1.2 \pm 0.4$ & $<0.001$ \\
\hline LDL-cholesterol (mmol/l) & $2.7 \pm 0.9$ & $3.9 \pm 1.4$ & $<0.001$ \\
\hline FBG $(\mathrm{mmol} / \mathrm{l})$ & $4.6 \pm 0.7$ & $4.9 \pm 0.9$ & 0.057 \\
\hline
\end{tabular}

Categorical data are presented as a proportion with corresponding percentages in parenthesis. Continuous variables are presented as mean \pm standard deviation. The proportions were compared using Fischer's exact test. Continuous variables were compared using independent t-test and Mann Whitney $\mathrm{U}$ test where applicable. Mets: HS: hypertensive strokes, WC: waist circumference, HC: hip circumference, WHR: waist to hip ratio, BMI: body mass index, SBP: systolic blood pressure, DBP: diastolic blood pressure, PP: pulse pressure, HTN: hypertension, CVD: cardiovascular disease, ARR: aldosterone renin ratio, HDL: high density lipoprotein, LDL: low density lipoprotein, FBG: fasting blood glucose. 
correlated with WC in non-metabolic syndrome subjects $(\mathrm{r}=0.274, p>0.05)$, as indicated in Table 5. Moreover, serum aldosterone negatively and significantly correlated with HDL-cholesterol $(r=-0.317, p<0.05)$ in metabolic syndrome subjects but was insignificant in non-metabolic syndrome subjects $(r=-0.429$, $p>0.05)$. In addition, serum aldosterone was significantly associated with increasing triglyceride in the metabolic syndrome group $(\mathrm{r}=0.439, p<0.05)$ but not in the non-metabolic syndrome group $(r=0.225, p>0.05)$.

\section{Discussion}

Metabolic syndrome (Metsyn) is known to increase the risk of adverse outcomes in stroke patients [24] [25]. Renin and aldosterone have been associated with the pathogenesis of obesity-related hypertension and weight loss has been shown to be complemented by reductions in plasma renin activity (PRA) and plasma aldosterone [26]. Albeit the role of the renin angiotensin aldosterone system in blood pressure regulation is well understood, its role in metabolic syndrome remains vague. In this study therefore, the relationships between the RAAS parameters and metabolic syndrome in some ischaemic stroke subjects were evaluated against data from apparently healthy cohorts within the same geographical boundary.

The study considered three different diagnostic criteria, NCEP, IDF and HSM. The overall degree of agreements between NCEP/IDF [bias $=0.107(-0.545-$ $0.760), \mathrm{r}=0.124(-0.043-0.284)$ ], NCEP/HMS [bias $=0.093(-0.525-0.710), \mathrm{r}$ $=0.118(-0.049-0.278)]$ and IDF/H_MS [bias $=-0.014(-0.346-0.317), \mathrm{r}=$ $-0.022(-0.187-0.145)]$ were excellent. However, each criteria gave different prevalence rates of $50.0 \%, 71.2 \%$ and $71.2 \%$ in the ischaemic group, $8.3 \%, 9.2 \%$, and $6.7 \%$ in the normotensive control group per the NCEP-ATP, IDF and HMS criteria, respectively (Table 2 and Table 3 ).

In this study, MetSyn prevalence in the ischaemic stroke subjects defined by IDF and HMS criteria are higher than in previous studies conducted among Chinese acute stroke subjects (51.35\%), ischaemic stroke subjects in India (60\%) and Iran (62\%) [6] [10] [27]. The discrepancies could be due to the differences in proportions of co-morbidities among study subjects. For instance, Mi et al. (2012) reported $87.5 \%$ hypertension prevalence in his subjects whereas $90.9 \%$ of the stroke subjects in this study were hypertensives. Sample size differences and inconsistencies in metabolic syndrome criteria could also have contributed to the differences in MetSyn prevalence.

The stroke subjects had significantly higher plasma renin, aldosterone and ARR compared to the control cohort (Table 2) suggesting that, the RAAS could have significant effect on cerebral circulation. In a review by [28], angiotensin peptides and angiotensinergic neural pathways were found to be important in neural function. Though the distribution of angiotensin II receptor 1 (AT 1) coincides with some brain regions, neither renin nor angiotensin II and its related peptides nor renin pass readily from the blood into the brain interstitium [29]. Local intrinsic RAAS has therefore been proposed [30]. 
Table 3. Prevalence of clustering of one or two or more components of metabolic syndrome.

\begin{tabular}{ccccc}
\hline PARAMETERS & Total & Controls & Cases & $p$-value \\
\hline NCEP-ATP & & & & \\
0 & $35(13.9)$ & $31(25.8)$ & $4(3.0)$ & $<0.001$ \\
1 & $88(34.9)$ & $64(53.3)$ & $24(18.2)$ & $<0.001$ \\
2 & $53(21.0)$ & $15(12.5)$ & $38(28.8)$ & 0.0012 \\
$\geq 3$ (MetSyn) & $76(30.2)$ & $10(8.3)$ & $66(50.0)$ & $<0.001$ \\
IDF, & & & & 0.060 \\
0 & $9(3.6)$ & $9(7.5)$ & $0(0.0)$ & $<0.001$ \\
1 & $90(37.7)$ & $68(56.7)$ & $22(16.7)$ & 0.035 \\
2 & $48(19.0)$ & $32(26.7)$ & $16(12.1)$ & $<0.001$ \\
$\geq 3$ (MetSyn) & $105(41.7)$ & $11(9.2)$ & $94(71.2)$ & \\
HMS, & & & & 0.014 \\
0 & $12(4.8)$ & $12(9.5)$ & $0(0.0)$ & $<0.001$ \\
1 & $91(36.1)$ & $71(59.5)$ & $20(15.2)$ & 0.135 \\
2 & $47(18.7)$ & $29(24.3)$ & $18(13.6)$ & $<0.001$ \\
\hline 3 (MetSyn) & $102(40.5)$ & $8(6.7)$ & $94(71.2)$ & \\
\hline
\end{tabular}

Data are presented as a proportion with corresponding percentages in parenthesis. The proportions were compared using Fischer's exact test. Mets: Metabolic Syndrome. HS: hypertensives with stroke, WHO: World Health Organization, NCEP-ATP III: National Cholesterol Education Program-Adult Treatment Panel III, IDF: International Diabetes Federation and H_MS: Harmonization.

This is apparent considering the fact that, anti-RAAS therapies have been proven to have cerebrovascular protective effects [31] [32].

Moreover, high serum aldosterone was independently associated with metabolic syndromein this study (Table 4). To the best of our knowledge, this is perhaps the first time RAAS and its role in MetSyn has been studied specifically in stroke subjects. The effect of aldosterone on MetSyn in other conditions has however been studied. Higher significant levels were found in MetSyn participants compared to non-MetSyn subjects among patients with obstructive sleep apnea hypopnea syndrome (OSAHS) [33]. Similarly, a cross-sectional study conducted in 356 participants from 69 families of African descent Seychelles found aldosterone to be independently associated with metabolic syndrome [34]. Furthermore, Kidambi et al., (2007) found that plasma aldosterone levels and not PRA, were elevated in subjects with metabolic syndrome $(P=0.0002)$ in a study conducted in 182 hypertensive cases and 215 normotensive control Black Americans [26]. Besides increasing blood pressure, non-genomic effects of mineralocorticoid receptor (MR) activation by aldosterone has been found to also include activation of mitogen-activated protein (MAP) kinase and intercellular adhesion molecule (ICAM) 1 pathways which play key roles in atherosclerosis [35]. Aldosterone also increases the production of reactive oxygen species (ROS) through decreases in glucose-6-phosphate dehydrogenase activity and activation 
Table 4. Predictors of metabolic syndrome in ischaemic strokes subjects.

\begin{tabular}{|c|c|c|c|c|c|c|}
\hline \multirow{2}{*}{ Variables } & \multicolumn{2}{|c|}{ Model A } & \multicolumn{2}{|c|}{ Model B } & \multicolumn{2}{|c|}{ Model C } \\
\hline & cOR $(95 \% \mathrm{CI})$ & $P$-value & aOR $(95 \% \mathrm{CI})$ & $P$-value & aOR $(95 \% \mathrm{CI})$ & $P$-value \\
\hline \multicolumn{7}{|l|}{ PAge } \\
\hline$<40$ & 1 & & 1 & & 1 & \\
\hline $40-60$ & $0.1(0.0-0.3)$ & 0.001 & $0.3(0.1-0.7)$ & 0.047 & $0.6(0.3-2.6)$ & 0.513 \\
\hline$>60$ & $0.3(0.1-1.7)$ & 0.177 & $0.5(0.2-1.8)$ & 0.235 & $0.7(0.3-3.2)$ & 0.723 \\
\hline Gender, males & $0.7(0.4-1.5)$ & 0.412 & $0.6(0.2-3.4)$ & 0.672 & $0.8(0.2-4.0)$ & 0.508 \\
\hline Alcohol status (yes) & $1.4(0.7-3.0)$ & 0.392 & $1.02(0.51-1.1)$ & 0.542 & $1.0(0.2-2.1)$ & 0.672 \\
\hline Smoking status (yes) & $2.3(0.5-4.9)$ & 0.413 & $1.8(0.6-3.3)$ & 0.444 & $1.5(0.6-3.0)$ & 0.700 \\
\hline FH of CVD (yes) & $1.8(0.24-2.5)$ & 0.672 & $1.09(0.00-3.27)$ & 0.093 & $1.1(0.0-2.9)$ & 0.087 \\
\hline Diabetes (yes) & $5.8(1.4-37.1)$ & $<0.001$ & $4.2(2.3-20.1)$ & 0.022 & $3.2(1.8-15.8)$ & 0.059 \\
\hline High BP ( $\geq 130 / 90$ mmHg) (yes) & $9.2(2.6-32.9)$ & 0.001 & $6.9(1.8-26.7)$ & 0.005 & $5.0(2.0-27.0)$ & 0.031 \\
\hline \multicolumn{7}{|l|}{ BMI $\left(\mathrm{kg} / \mathrm{m}^{2}\right)$} \\
\hline Overweight & $2.5(1.1-5.6)$ & 0.032 & $3.8(1.5-9.5)$ & 0.140 & $2.8(0.7-11.0)$ & 0.140 \\
\hline Obese & $12.5(4.0-38.6)$ & $<0.001$ & $18.5(5.2-66.5)$ & 0.004 & $11.7(2.2-62.8)$ & 0.004 \\
\hline \multicolumn{7}{|l|}{ Plasma Renin } \\
\hline $\mathrm{T}_{2}(25.0-38.2) \mathrm{pg} / \mathrm{ml}$ & $8.5(1.0-72.0)$ & 0.049 & $8.3(1.0-71.5)$ & 0.053 & $4.0(0.4-38.8)$ & 0.228 \\
\hline $\mathrm{T}_{3}(>38.2) \mathrm{pg} / \mathrm{ml}$ & $9.1(7.6-59.8)$ & $<0.001$ & $6.4(4.2-40.5)$ & $<0.001$ & $1.6(0.1-22.7)$ & 0.742 \\
\hline \multicolumn{7}{|l|}{ Plasma Aldosterone } \\
\hline $\mathrm{T}_{2}(182-504) \mathrm{pg} / \mathrm{ml}$ & $2.1(0.4-12.5)$ & 0.402 & $2.3(0.4-13.7)$ & 0.357 & $2.0(0.3-13.8)$ & 0.478 \\
\hline $\mathrm{T}_{3}(>504) \mathrm{pg} / \mathrm{ml}$ & $3.6(1.1-43.2$ & $<0.001$ & $3.1(1.8-41.5)$ & $<0.001$ & $2.7(1.2-10.0)$ & 0.008 \\
\hline \multicolumn{7}{|l|}{ Total Cholesterol (mmol/l) } \\
\hline borderline high $(5.18-6.18)$ & $0.3(0.1-0.9)$ & 0.034 & $0.4(0.1-1.0)$ & 0.057 & $0.3(0.1-1.6)$ & 0.156 \\
\hline High $(\geq 6.12)$ & $2.4(1.1-5.6)$ & 0.039 & $2.5(1.1-6.0)$ & 0.033 & $1.3(0.3-5.0)$ & 0.708 \\
\hline \multicolumn{7}{|l|}{ Triglyceride (mmol/l) } \\
\hline Borderline high (1.7 - 2.2) & $0.1(0.0-1.2)$ & 0.077 & $0.1(0.0-0.9)$ & $0.044)$ & $3.4(0.3-45.0)$ & 0.360 \\
\hline High $(>2.2)$ & $5.5(1.7-18.3)$ & 0.005 & $4.5(1.3-15.5)$ & 0.017 & $5.3(4.0-314.0)$ & $<0.001$ \\
\hline \multicolumn{7}{|l|}{ HDL cholesterol (mmol/l) } \\
\hline Medium (1.0 - 1.6) & $0.1(0.0-1.4)$ & 0.115 & $0.6(0.3-2.2)$ & 0.300 & $0.8(0.2-9.3)$ & 0.414 \\
\hline High $(>1.6)$ & $0.4(0.0-0.8)$ & $<0.001$ & $0.6(0.2-3.5)$ & 0.205 & $0.5(0.0-3.0)$ & 0.431 \\
\hline LDL-C $(>4.13) \mathrm{mmol} / 1$ & $0.8(0.2-2.7)$ & 0.735 & $1.1(0.3-3.9)$ & 0.874 & $1.0(0.2-4.0)$ & 0.937 \\
\hline
\end{tabular}

Model A: unadjusted; Model B: adjusted for age and gender; Model C: adjusted for age, gender, diabetes and hypertension; Reference categories: Age ( $<40$ years), healthy BMI $\left(18.5-25.0 \mathrm{~kg} / \mathrm{m}^{2}\right)$, first tertile renin $(<25.0 \mathrm{pg} / \mathrm{ml})$, first tertile aldosterone $(>504.0 \mathrm{pg} / \mathrm{ml})$, desirable total cholesterol $(<5.18 \mathrm{mmol} / \mathrm{l})$, desirable triglyceride $(<1.7 \mathrm{mmol} / \mathrm{l})$, low HDL cholesterol $(<1.0 \mathrm{mmol} / \mathrm{l})$ and optimal LDL cholesterol $(<2.59 \mathrm{mmol} / \mathrm{l})$.

of NAD (P) $\mathrm{H}$ oxidase [36]. Mineralocorticoid receptor antagonists used in combination with ACE inhibitors and or angiotensin II blockers (ARBs) have shown beneficial effects in diabetic nephropathy [37].

MR antagonists may therefore have positive effects on various components of 
the MetSyn through improving insulin sensitivity, reducing blood pressure, decreasing the pro-inflammatory state of MetSyn, and hence reduction in CVD occurrences [12].

Aldosterone significantly correlated negatively with HDL-cholesterol $(\mathrm{r}=$ $-0.317, p<0.05)$ and positively with triglyceride concentration $(\mathrm{r}=0.439, p<$ 0.05 ) in the metabolic syndrome participants (Table 5). Though the mechanism behind these correlations is not fully understood, aldosterone production has been shown to be stimulated by free fatty acids released from visceral adipose tissues [38] [39]. This is also consistent with the observation that drospirenone, an oral contraceptive with antimineralocorticoid properties, which is chemically related to spironolactone (a mineralocorticoid receptor blocker), significantly increased HDL cholesterol in young healthy women [40].

In this study, increasing plasma renin was not independently associated with metabolic syndrome (Table 4). Contrary to this, among 29 volunteers with a range of risk factors studied, patients with more metabolic syndrome factor clustering including abdominal obesity and high blood pressure had significantly greater values for renin independent of dietary salt intake [41]. More recently, a prospective study involving 5301 African American adults, aged 21 - 94 years showed that log-transformed plasma renin levels were associated with Metsyn and its components in fully adjusted models [42].

In this study, plasma renin levels significantly correlated negatively $(\mathrm{r}=$ $-0.319, p<0.05)$ with HDL-cholesterol levels. Concordance with this study, multivariate logistic regression analysis in a cross-sectional study showed that high PRA was independently associated high HDL-C [43]. Another cross-sectional study in 985 subjects with metSyn and 515 without metSyn showed that renin levels increased and correlated inversely with HDL [44]. Conversely, in a

Table 5. Correlation co-efficient between Renin, Aldosterone and some metabolic Parameters in the hypertensive stroke subjects.

\begin{tabular}{ccccc}
\hline \multirow{2}{*}{ Parameters } & \multicolumn{2}{c}{ Non-MetSyn } & \multicolumn{2}{c}{ MetSyn } \\
\cline { 2 - 5 } & Renin $(\mathrm{pg} / \mathrm{ml})$ & Aldosterone $(\mathrm{pg} / \mathrm{ml})$ & Renin $(\mathrm{pg} / \mathrm{ml})$ & Aldosterone $(\mathrm{pg} / \mathrm{ml})$ \\
\hline WC & 0.274 & $0.515^{\star}$ & $0.493^{\star}$ & $0.588^{*}$ \\
HC & 0.195 & 0.453 & 0.139 & 0.262 \\
WHR & 0.422 & 0.457 & 0.511 & $0.503^{*}$ \\
BMI & 0.202 & $0.667^{*}$ & 0.260 & $0.691^{*}$ \\
HDL & $-0.608^{*}$ & -0.429 & $-0.319^{*}$ & $-0.317^{*}$ \\
LDL & $0.579^{*}$ & $0.546^{*}$ & 0.393 & $0.318^{*}$ \\
TC & $0.466^{*}$ & $0.403^{*}$ & 0.371 & $0.678^{\star}$ \\
FBG & 0.213 & 0.176 & 0.281 & 0.492 \\
TG & 0.446 & 0.225 & 0.481 & $0.439^{*}$ \\
\hline
\end{tabular}

*significant correlation $(p<0.05)$; WC, waist circumference; HC, hip circumference; WHR, waist-to-hip ratio; BMI, body mass index; HDL, high density lipoprotein; LDL, low density lipoprotein; TC, total cholesterol; FBG, fasting blood glucose; TG, triglyceride. 
cross-sectional study involving 80 males with central obesity, plasma renin activity had a positive correlation with HDL-cholesterol $(r=0.273 ; p=0.014)$ [45]. The effects of anti-hypertensive drugs could be the reason behind the contrary views expressed.

We also witnessed a positive significant correlation between plasma renin and waist circumference $(r=0.493, p<0.05)$. This is suggestive of renin playing key role in obesity and is in keeping with increased plasma angiotensinogen (a direct product of renin activity) in obese patients as compared with non-obese patients in a study by [46]. Moreover, an in vivo investigation of angiotensinogen (AGT) expression in adipose tissue and liver of Zucker rats during the onset of obesity showed adipocytes from obese rats displaying a significant increase in AGT content than in non-obese rats [47].

Increased renin activity, a characteristic of an up regulated renin-angiotensinaldosterone system has been described in patients with visceral obesity which improves with weight loss [9]. This has been explained by many as a result of the presence of local renin-angiotensin-aldosterone system in the adipose tissue which may play a significant role in the pathophysiology of obesity and of obesity-related hypertension [11] [48].

Whilst a case can be made about the potential role of both renin and aldosterone in the pathophysiology of metabolic syndrome among ischaemic stroke patients in this study, the use of case-control study design limits their absolute cause and effect relationship. Moreover, plasma renin was measured as a concentration other than the more standardized measurement of its activity that is plasma renin activity (PRA). Therefore, further studies which employ robust study design involving follow-ups and measurement of PRA instead of concentration are warranted to fully understand the mechanism behind the RAAS and metabolic syndrome.

\section{Conclusion}

This study has demonstrated a high prevalence of metabolic syndrome among ischaemic stroke patients at the Komfo Anokye Teaching Hospital. Raised blood aldosterone was shown to be significantly associated with metabolic syndrome in the stroke subjects. These findings underpin the observations that increased aldosterone levels may exacerbate metabolic defects in people with ischaemic stroke. Therefore, combination of standard treatments with available mineralocorticoid receptor antagonists including spironolactone and eplerenone in ischaemic strokes may halt further vascular deterioration and re-occurrence of stroke. Other potent risk factors identified were high blood pressure, obesity and triglyceridaemia. Further studies with larger sample size and robust study design involving follow ups are warranted to fully understand the role RAAS plays in cardio-metabolic syndrome in ischaemic strokes.

\section{Acknowledgements}

The authors would like to acknowledge the management and staff of Department 
of Medicine, Komfo Anokye Teaching Hospital for allowing us carry out this work in their department. We are also grateful to all participants of this work for voluntarily agreeing to partake in this study.

\section{Competing Interest}

The authors declare that there is no conflict of interest regarding the publication of this paper.

\section{Funding}

There was no funding for this work

\section{Ethical Approval}

Ethical approval (CHRPE/AP/377/15) for the study was obtained from the Committee on Human Research, Publication and Ethics (CHRPE) of the School of Medical Sciences (SMS), Kwame Nkrumah University of Science and Technology (KNUST) and Research and Development Unit KATH. Informed consent was obtained from all participants and participation was voluntary.

\section{Contributorship}

Francis Agyemang Yeboah contributed to the conception of the research idea, design data analysis and interpretation, paper drafting and revision. Benjamin Ackon Eghan contributed to the conception of the research idea, design, data analysis and interpretation, paper drafting and revision. Bernard Nkum contributed to the conception of the research idea, design, data collection, data analysis and interpretation, paper drafting and revision

Bright Amankwaa contributed to research design, patient recruitment, collection data analysis and interpretation, paper drafting and revision. Emmanuel Acheampong contributed to research design, patient recruitment and collection. Paul Nsiah contributed to the conception of the research idea, design and data collection. Lawrence Owusu and Perditer Okyere contributed to the conception of the research idea, design, and data collection. Emmanuella Batu Nsenba contributed to the research idea, design and data collection. Enoch Odame Anto contributed to research design, patient recruitment, collection data analysis and interpretation.

\section{References}

[1] Boldyreff, B. and Wehling, M. (2003) Non-Genomic Actions of Aldosterone: Mechanisms and Consequences in Kidney Cells. Nephrology Dialysis Transplantation, 18, 1693-1695. https://doi.org/10.1093/ndt/gfg265

[2] Alberti, K.G.M., Zimmet, P. and Shaw, J. (2005) The Metabolic Syndrome-A New Worldwide Definition. The Lancet, 366, 1059-1062.

https://doi.org/10.1016/S0140-6736(05)67402-8

[3] Wang, C.-H., Li, F. and Takahashi, N. (2010) The Renin Angiotensin System and the Metabolic Syndrome. The Open Hypertension Journal, 3, 1-13. 
https://doi.org/10.2174/1876526201003010001

[4] Abdou, M., AbdElGawad, M.S. and Mohamed, S.A. (2013) Increased Aldosterone in Patients with Metabolic Syndrome: An Additional Risk Factor. Journal of Diabetes Research and Clinical Metabolism, 2, 17.

[5] Kaur, J. (2014) A Comprehensive Review on Metabolic Syndrome. Cardiology Research and Practice, 2014, 943162.

[6] Mathew, B. (2014) Incidence of Metabolic Syndrome in Ischemic Stroke Patients in South Indian Population. International Journal of Pharmaceutical and Phytopharmacological Research, 3, 110-113.

[7] Akpalu, J., Akpalu, A. and Ofei, F. (2011) The Metabolic Syndrome among Patients with Cardiovascular Disease in Accra, Ghana. Ghana Medical Journal, 45, 161-166.

[8] Stiefel, P., Vallejo-Vaz, A., García Morillo, S. and Villar, J. (2011) Role of the Renin-Angiotensin System and Aldosterone on Cardiometabolic Syndrome. International Journal of Hypertension, 2011, 685238.

[9] Engeli, S. (2006) Role of the Renin-Angiotensin-Aldosterone System in the Metabolic Syndrome. In: Obesity and the Kidney, Volume 151, Karger Publishers, Basel, 122-134.

[10] Ashtari, F., Salari, M., Amminoroaya, A., Deljoo, B.K. and Moeini, M. (2012) Metabolic Syndrome in Ischemic Stroke: A Case-Control Study. Journal of Research in Medical Sciences, 17, 167-170.

[11] Sarzani, R., Salvi, F., Dessì-Fulgheri, P. and Rappelli, A. (2008) Renin-Angiotensin System, Natriuretic Peptides, Obesity, Metabolic Syndrome, and Hypertension: An Integrated View in Humans. Journal of Hypertension, 26, 831-843. https://doi.org/10.1097/HJH.0b013e3282f624a0

[12] Tirosh, A., Garg, R. and Adler, G.K. (2010) Mineralocorticoid Receptor Antagonists and the Metabolic Syndrome. Current Hypertension Reports, 12, 252-257. https://doi.org/10.1007/s11906-010-0126-2

[13] Guo, C., Ricchiuti, V., Lian, B.Q., Yao, T.M., Coutinho, P., Romero, J.R., Li, J., Williams, G.H. and Adler, G.K. (2008) Mineralocorticoid Receptor Blockade Reverses Obesity-Related Changes in Expression of Adiponectin, Peroxisome Proliferator-Activated Receptor- $\gamma$, and Proinflammatory Adipokines. Circulation, 117, 2253-2261. https://doi.org/10.1161/CIRCULATIONAHA.107.748640

[14] Vogt, B., Bochud, M. and Burnier, M. (2007) The Association of Aldosterone with Obesity-Related Hypertension and the Metabolic Syndrome. In: Seminars in Nephrology, Elsevier, 529-537.

[15] Agyemang, C., Attah-Adjepong, G., Owusu-Dabo, E., Aikins, A.D.G., Addo, J., Edusei, A., Nkum, B. and Ogedegbe, G. (2012) Stroke in Ashanti Region of Ghana. Ghana Medical Journal, 46, 12-17.

[16] Saiedullah, M., Sarkar, A., Kamaluddin, S.M., Begum, S., Hayat, S., Rahman, M.R. and Khan, M.A.H. (2011) Friedewald's Formula Is Applicable up to Serum Triacylglycerol to Total Cholesterol Ratio of Two in Bangladeshi Population. Anwer Khan Modern Medical College Journal, 2, 21-25.

[17] Owusu, I. (2007) Causes of Heart Failure as Seen in Kumasi, Ghana. The Internet Journal of Third World Medicine, 5, 201-214.

[18] Shen, J., Tryon, D., Myklak, K., Alsyouf, M., Peplinski, B., Conceicao, C., Ruckle, H., Baldwin, D., Liu, L. and Johnson, L. (2015) Scientific Program of 33rd World Congress of Endourology \& SWL Program Book. Journal of Endourology, 29, P1-A457. https://doi.org/10.1089/end.2015.29003.abstracts 
[19] Tomaschitz, A., Maerz, W., Pilz, S., Ritz, E., Scharnagl, H., Renner, W., Boehm, B.O., Fahrleitner-Pammer, A., Weihrauch, G. and Dobnig, H. (2010) Aldosterone/Renin Ratio Determines Peripheral and Central Blood Pressure Values over a Broad Range. Journal of the American College of Cardiology, 55, 2171-2180.

[20] Stevens, L.A., Schmid, C.H., Greene, T., Zhang, Y.L., Beck, G.J., Froissart, M., Hamm, L.L., Lewis, J.B., Mauer, M. and Navis, G.J. (2010) Comparative Performance of the CKD Epidemiology Collaboration (CKD-EPI) and the Modification of Diet in Renal Disease (MDRD) Study Equations for Estimating GFR Levels above $60 \mathrm{~mL} / \mathrm{min} / 1.73 \mathrm{~m}$ 2. American Journal of Kidney Diseases, 56, 486-495. https://doi.org/10.1053/j.ajkd.2010.03.026

[21] Health NIO, National Heart L, Institute B (2001) ATP III Guidelines at-a-glance Quick Desk Reference. NIH Publication (01-3305).

[22] Alberti, K.G.M.M., Zimmet, P. and Shaw, J. (2006) Metabolic Syndrome-A New World-Wide Definition. A Consensus Statement from the International Diabetes Federation. Diabetic Medicine, 23, 469-480. https://doi.org/10.1111/j.1464-5491.2006.01858.x

[23] Alberti, K., Eckel, R.H., Grundy, S.M., Zimmet, P.Z., Cleeman, J.I., Donato, K.A., Fruchart, J.-C., James, W.P.T., Loria, C.M. and Smith, S.C. (2009) Harmonizing the Metabolic Syndrome. Circulation, 120, 1640-1645. https://doi.org/10.1161/CIRCULATIONAHA.109.192644

[24] Palomeras Soler, E. and Casado Ruiz, V. (2010) Epidemiology and Risk Factors of Cerebral Ischemia and Ischemic Heart Diseases: Similarities and Differences. Current Cardiology Reviews, 6, 138-149. https://doi.org/10.2174/157340310791658785

[25] Protack, C.D., Bakken, A.M., Xu, J., Saad, W.A., Lumsden, A.B. and Davies, M.G. (2009) Metabolic Syndrome: A Predictor of Adverse Outcomes after Carotid Revascularization. Journal of Vascular Surgery, 49, 1172-1180.

[26] Kidambi, S., Kotchen, J.M., Grim, C.E., Raff, H., Mao, J., Singh, R.J. and Kotchen, T.A. (2007) Association of Adrenal Steroids with Hypertension and the Metabolic Syndrome in Blacks. Hypertension, 49, 704-711. https://doi.org/10.1161/01.HYP.0000253258.36141.c7

[27] Mi, D., Jia, Q., Zheng, H., Hoff, K., Zhao, X., Wang, C., Liu, G., Wang, Y., Liu, L. and Wang, X. (2012) Metabolic Syndrome and Stroke Recurrence in Chinese Ischemic Stroke Patients-The ACROSS-China Study. PLoS ONE, 7, e51406. https://doi.org/10.1371/journal.pone.0051406

[28] McKinley, M., Albiston, A., Allen, A., Mathai, M., May, C., McAllen, R., Oldfield, B., Mendelsohn, F. and Chai, S. (2003) The Brain Renin-Angiotensin System: Location and Physiological Roles. The International Journal of Biochemistry \& Cell Biology, 35, 901-918.

[29] Allen, A., Oldfield, B., Giles, M., Paxinos, G., McKinley, M. and Mendelsohn, F. (2000) Chapter III Localization of Angiotensin Receptors in the Nervous System. Handbook of Chemical Neuroanatomy, 16, 79-124.

[30] Baltatu, O.C., Campos, L.A. and Bader, M. (2011) Local Renin-Angiotensin System and the Brain-A Continuous Quest for Knowledge. Peptides, 32, 1083-1086.

[31] MacLeod, A.B., Vasdev, S. and Smeda, J.S. (1997) The Role of Blood Pressure and Aldosterone in the Production of Hemorrhagic Stroke in Captopril-Treated Hypertensive Rats. Stroke, 28, 1821-1829. https://doi.org/10.1161/01.STR.28.9.1821

[32] Atkinson, J. (2011) Stroke, High Blood Pressure and the Renin-Angiotensin Aldosterone System New Developments. Frontiers in Pharmacology, 2, 22. 
[33] Barceló, A., Piérola, J., Esquinas, C., de la Peña, M., Arqué, M., Alonso-Fernández, A., Bauçà, J.M., Robles, J., Barceló, B. and Barbé, F. (2014) Relationship between Aldosterone and the Metabolic Syndrome in Patients with Obstructive Sleep Apnea Hypopnea Syndrome: Effect of Continuous Positive Airway Pressure Treatment. PLOS ONE, 9, e84362. https://doi.org/10.1371/journal.pone.0084362

[34] Bochud, M., Nussberger, J., Bovet, P., Maillard, M.R., Elston, R.C., Paccaud, F., Shamlaye, C. and Burnier, M. (2006) Plasma Aldosterone Is Independently Associated with the Metabolic Syndrome. Hypertension, 48, 239-245. https://doi.org/10.1161/01.HYP.0000231338.41548.fc

[35] Grossmann, C. and Gekle, M. (2009) New Aspects of Rapid Aldosterone Signaling. Molecular and Cellular Endocrinology, 308, 53-62.

[36] Sun, Y., Zhang, J., Lu, L., Chen, S.S., Quinn, M.T. and Weber, K.T. (2002) Aldosterone-Induced Inflammation in the Rat Heart: Role of Oxidative Stress. The American Journal of Pathology, 161, 1773-1781.

[37] Sato, A., Hayashi, K. and Saruta, T. (2005) Antiproteinuric Effects of Mineralocorticoid Receptor Blockade in Patients with Chronic Renal Disease. American Journal of Hypertension, 18, 44-49.

[38] Goodfriend, T., Egan, B. and Kelley, D. (1999) Plasma Aldosterone, Plasma Lipoproteins, Obesity and Insulin Resistance in Humans. Prostaglandins, Leukotrienes and Essential Fatty Acids, 60, 401-405.

[39] Goodfriend, T.L., Ball, D.L., Egan, B.M., Campbell, W.B. and Nithipatikom, K. (2004) Epoxy-Keto Derivative of Linoleic Acid Stimulates Aldosterone Secretion. Hypertension, 43, 358-363. https://doi.org/10.1161/01.HYP.0000113294.06704.64

[40] Oelkers, W., Foidart, J.-M., Dombrovicz, N., Welter, A. and Heithecker, R. (1995) Effects of a New Oral Contraceptive Containing an Antimineralocorticoid Progestogen, Drospirenone, on the Renin-Aldosterone System, Body Weight, Blood Pressure, Glucose Tolerance, and Lipid Metabolism. The Journal of Clinical Endocrinology \& Metabolism, 80, 1816-1821.

[41] Egan, B.M., Stepniakowski, K. and Goodfriend, T.L. (1994) Renin and Aldosterone Are Higher and the Hyperinsulinemic Effect of Salt Restriction Greater in Subjects with Risk Factors Clustering. American Journal of Hypertension, 7, 886-893. https://doi.org/10.1093/ajh/7.10.886

[42] Joseph, J.J., Echouffo-Tcheugui, J.B., Kalyani, R.R., Yeh, H.-C., Bertoni, A.G., Effoe, V.S., Casanova, R., Sims, M., Correa, A. and Wu, W.-C. (2016) Aldosterone, Renin, and Diabetes Mellitus in African Americans: The Jackson Heart Study. The Journal of Clinical Endocrinology \& Metabolism, 101, 1770-1778.

https://doi.org/10.1210/jc.2016-1002

[43] Abiko, H., Konta, T., Hao, Z., Takasaki, S., Suzuki, K., Ichikawa, K., Ikeda, A., Shibata, Y., Takeishi, Y. and Kawata, S. (2009) Factors Correlated with Plasma Renin Activity in General Japanese Population. Clinical and Experimental Nephrology, 13, 130. https://doi.org/10.1007/s10157-008-0114-x

[44] Gonçalves, I., Edsfeldt, A., Colhoun, H.M., Shore, A.C., Palombo, C., Natali, A., Fredrikson, G.N., Björkbacka, H., Wigren, M. and Bengtsson, E. (2016) Association between Renin and Atherosclerotic Burden in Subjects with and without Type 2 Diabetes. BMC Cardiovascular Disorders, 16, 171.

https://doi.org/10.1186/s12872-016-0346-8

[45] Intantri, H.Y., Wijaya, A. and Patellongi, I. (2011) Association of Aldosterone, Plasma Renin Activity (PRA) and Superoxide Dismutase (SOD) with Inflammation and Insulin Resistance in Adult Men with Central Obesity. The Indonesian Biomedical Journal, 3, 116-121. https://doi.org/10.18585/inabj.v3i2.141 
[46] Umemura, S., Nyui, N., Tamura, K., Hibi, K., Yamaguchi, S., Nakamaru, M., Ishigami, T., Yabana, M., Kihara, M. and Inoue, S. (1997) Plasma Angiotensinogen Concentrations in Obese Patients. American Journal of Hypertension, 10, 629-633.

[47] Hainault, I., Nebout, G., Turban, S., Ardouin, B., Ferré, P. and Quignard-Boulangé, A. (2002) Adipose Tissue-Specific Increase in Angiotensinogen Expression and Secretion in the Obese (fa/fa) Zucker Rat. American Journal of Physiology-Endocrinology and Metabolism, 282, E59-E66.

[48] Engeli, S., Böhnke, J., Gorzelniak, K., Janke, J., Schling, P., Bader, M., Luft, F.C. and Sharma, A.M. (2005) Weight Loss and the Renin-Angiotensin-Aldosterone System. Hypertension, 45, 356-362. https://doi.org/10.1161/01.HYP.0000154361.47683.d3

Submit or recommend next manuscript to OALib Journal and we will provide best service for you:

- Publication frequency: Monthly

- 9 subject areas of science, technology and medicine

- Fair and rigorous peer-review system

- Fast publication process

- Article promotion in various social networking sites (LinkedIn, Facebook, Twitter, etc.)

- Maximum dissemination of your research work

Submit Your Paper Online: Click Here to Submit

Or Contact service@oalib.com 